Effect of straining graphene on nanopore creation using Si cluster bombardment: A reactive atomistic investigation

G. R. Berdiyorov, B. Mortazavi, S. Ahzi, F. M. Peeters, and M. K. Khraisheh

Citation: J. Appl. Phys. 120, 225108 (2016); doi: 10.1063/1.4971767

View online: http://dx.doi.org/10.1063/1.4971767

View Table of Contents: http://aip.scitation.org/toc/jap/120/22

Published by the American Institute of Physics

Articles you may be interested in

Anisotropic control of thermal transport in graphene/Si heterostructures

J. Appl. Phys. 120, 225111 (2016); 10.1063/1.4971873 


\title{
Effect of straining graphene on nanopore creation using Si cluster bombardment: A reactive atomistic investigation
}

\author{
G. R. Berdiyorov, ${ }^{1, a)}$ B. Mortazavi, ${ }^{2}$ S. Ahzi, ${ }^{1}$ F. M. Peeters, ${ }^{3}$ and M. K. Khraisheh ${ }^{1}$ \\ ${ }^{1}$ Qatar Environment and Energy Research Institute, Hamad Bin Khalifa University, Doha, Qatar \\ ${ }^{2}$ Bauhaus-Universität Weimar, Marienstr. 15, D-99423 Weimar, Germany \\ ${ }^{3}$ Departement Fysica, Universiteit Antwerpen, Groenenborgerlaan 171, B-2020 Antwerpen, Belgium
}

(Received 22 August 2016; accepted 23 November 2016; published online 15 December 2016)

\begin{abstract}
Graphene nanosheets have recently received a revival of interest as a new class of ultrathin, high-flux, and energy-efficient sieving membranes because of their unique two-dimensional and atomically thin structure, good flexibility, and outstanding mechanical properties. However, for practical applications of graphene for advanced water purification and desalination technologies, the creation of well controlled, high-density, and subnanometer diameter pores becomes a key factor. Here, we conduct reactive force-field molecular dynamics simulations to study the effect of external strain on nanopore creation in the suspended graphene by bombardment with Si clusters. Depending on the size and energy of the clusters, different kinds of topography were observed in the graphene sheet. In all the considered conditions, tensile strain results in the creation of nanopores with regular shape and smooth edges. On the contrary, compressive strain increases the elastic response of graphene to irradiation that leads to the formation of net-like defective structures with predominantly carbon atom chains. Our findings show the possibility of creating controlled nanopores in strained graphene by bombardment with Si clusters. Published by AIP Publishing.

[http://dx.doi.org/10.1063/1.4971767]
\end{abstract}

\section{INTRODUCTION}

Since the first mechanical exfoliation of graphene from graphite, an enormous interest appeared to explore applications of graphene in different fields such as electronics, photonics, sensors, and catalysis. ${ }^{1-6}$ Recently, graphene nanosheets have also emerged as a new class of ultrathin, high-flux and energyefficient sieving membranes for water desalination because of their unique two-dimensional and atomically thin structure, outstanding mechanical strength, and good flexibility. ${ }^{7-20}$ However, utilization of the full potential of graphene for advanced membrane technologies necessitates a fundamental understanding of the origin of the permeability of graphene and other carbon based materials. ${ }^{21}$ It is well known that pristine graphene is not permeable even for small gas atoms like helium. ${ }^{22}$ Therefore, in order to permeate through graphene membranes, water molecules should take a tortuous path through the structural defects of graphene sheets and in interconnected nanochannels formed between graphene nanosheets. ${ }^{8}$ To enhance the ion-selectivity of graphene based membranes, the size of the synthetic pores should be commensurable with the diameter of the translocating ions, and the pore walls should have the proper chemical residues to interact selectively with the ions (see Refs. 23-25 for reviews). Therefore, the controlled introduction and stabilization of nanoscale pores in graphene is crucial for the application of graphene in water treatment. ${ }^{26,27}$

Metal ion bombardment is considered to be one of the effective methods of creating nanopores in graphene ${ }^{28-32}$ The size, energy, and shooting angle of the metallic clusters can be

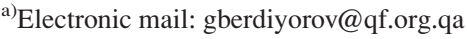

used as tuning parameters to control the size of the resulting nanopores. For example, Bai et al. showed that the nanopore family can be significantly expanded by using oblique ion beam irradiation to include different types of nanopores of tunable geometries. ${ }^{33}$ Depending on the energy of the ions and the incident angles, carbon atoms can be sputtered even outside the impact zone. Qin et al. conducted molecular dynamics (MD) simulations to study nanopore creation in graphene by silicon ions. ${ }^{30}$ They found that different processes can take place depending on the energy of ions such as absorption, replacement, transmission, and damage. Zhao et al. also used MD simulations to study nanopore creation processes in graphene using different metal ion clusters. ${ }^{29} \mathrm{Li}$ et al. conducted MD simulations to study the interaction of heavy ions using reactive force fields. ${ }^{32}$ Wang et al. performed experiments and created vacancy defects in graphene using metal ion bombardment. ${ }^{28}$ They also showed possibilities of doping graphene with different metal atoms. The size of the nanopores can further be tuned with an electron beam. ${ }^{34}$ Functionalization of the nanopore edges also plays an important role. For example, the selectivity of graphene to different kinds of ions (anions and cations) can be increased after nanopore edge passivation. ${ }^{35} \mathrm{In}$ addition, the passivation of dangling bonds can also stabilize the nanopores, as was shown in recent experiments by Lee et al. ${ }^{26}$ Desalination properties of perforated graphene can further be increased by forming multilayered structures. ${ }^{36}$

Here, we perform reactive force MD simulations to study the effect of external strain on nanopore creation in graphene by energetic Si clusters. We consider free standing graphene in order to avoid the effect of a substrate on the nanopore formation process. ${ }^{37-39}$ Silicon clusters are used for the bombardment motivated from the fact that $\mathrm{Si}$ atoms 
can enhance the stability of graphene nanopores against carbon filling. ${ }^{26}$ Depending on the size and energy of the clusters, three processes are active: reflection, absorption, and damage (i.e., nanopore creation). The elastic response of graphene to the irradiation increases with applying compressive strain, resulting in the formation of net-like defective structures with dangling carbon atom chains. The latter acts as reactive centers for chemical functionalization which affects the stability of the created nanopores. On the contrary, tensile strain results in the creation of nanopores with smoother edges. Our findings indicate the possibility of using external strain to introduce controlled, subnanometer-sized pores using bombardement with clusters.

\section{COMPUTATIONAL DETAILS AND CHARACTERIZATION}

MD simulations are conducted using the reactive forcefield (ReaxFF), which, in contrast to classical force-fields, is a general bond-order dependent potential that can account for dynamic dissociation and formation of chemical bonds during the simulations. ${ }^{40}$ The bonds connectivity in the entire system is recalculated at every simulation step, and the non-bounded interactions are considered by both van der Waals and Coulomb forces. Since ReaxFF parameters are derived from quantum chemical calculations, it gives transition states and reaction pathways in agreement with quantum mechanical calculations and experiments. ${ }^{40}$ ReaxFF force fields have already been successfully used in describing the properties of graphene with silicon inclusions. ${ }^{41}$

As a representative example, we consider free standing graphene with 5376 carbon atoms, corresponding to a computational unit cell of $118.13 \times 119.35 \AA^{2}$ in the $x-y$-plane. Periodic boundary conditions are used along the graphene basal plane in order to avoid edge effects, and a vacuum space of $200 \AA$ is applied along the $z$-direction. We first increased the temperature of the system up to $300 \mathrm{~K}$ at a rate of $6 \mathrm{~K} / \mathrm{ps}$ using an isothermal isobaric (NPT) ensemble with a Nose-Hoover thermostat/barostat for temperature/ pressure control. The damping constants for temperature and pressure were $100 \mathrm{fs}$ and $1 \mathrm{ps}$, respectively, and the time step was 0.25 fs. After such thermalization, we have conducted 25 ps long non-equilibrium MD simulations by continuously applying compressive (Fig. 1(a)) or tensile (Fig. 1(b)) strain to the system. The strained samples were further thermalized at $300 \mathrm{~K}$ for another $25 \mathrm{ps}$ using a canonical (NVT) ensemble with a damping time of $100 \mathrm{fs}$. Silicon clusters were initially equilibrated separately in a simulation box with size larger than $10 \AA$ using NVT ensemble (Fig. 1(c)). No explicit net charges are present on the considered clusters.

The thermalized Si clusters were then positioned at $50 \AA$ on top of the graphene, and different initial velocities were assigned to each cluster. The energies of the incident clusters were in the range between $3 \mathrm{eV} /$ atom and $350 \mathrm{eV} /$ atom, and the incident angle was chosen to be normal to graphene's basal plane. As typical examples, we consider Si clusters containing 6, 10, and $16 \mathrm{Si}$ atoms (see Fig. 1(c)). Simulations were conducted using a microcanonical (NVE) ensemble using a small time step of $0.1 \mathrm{fs}$, in order to track the impact phenomena with a higher resolution. After the impact, the system is relaxed for the next 200 ps. During this time, all the atoms leaving the system in the $z$-direction are lost. Due to the stochastic nature of damage formation during the metal
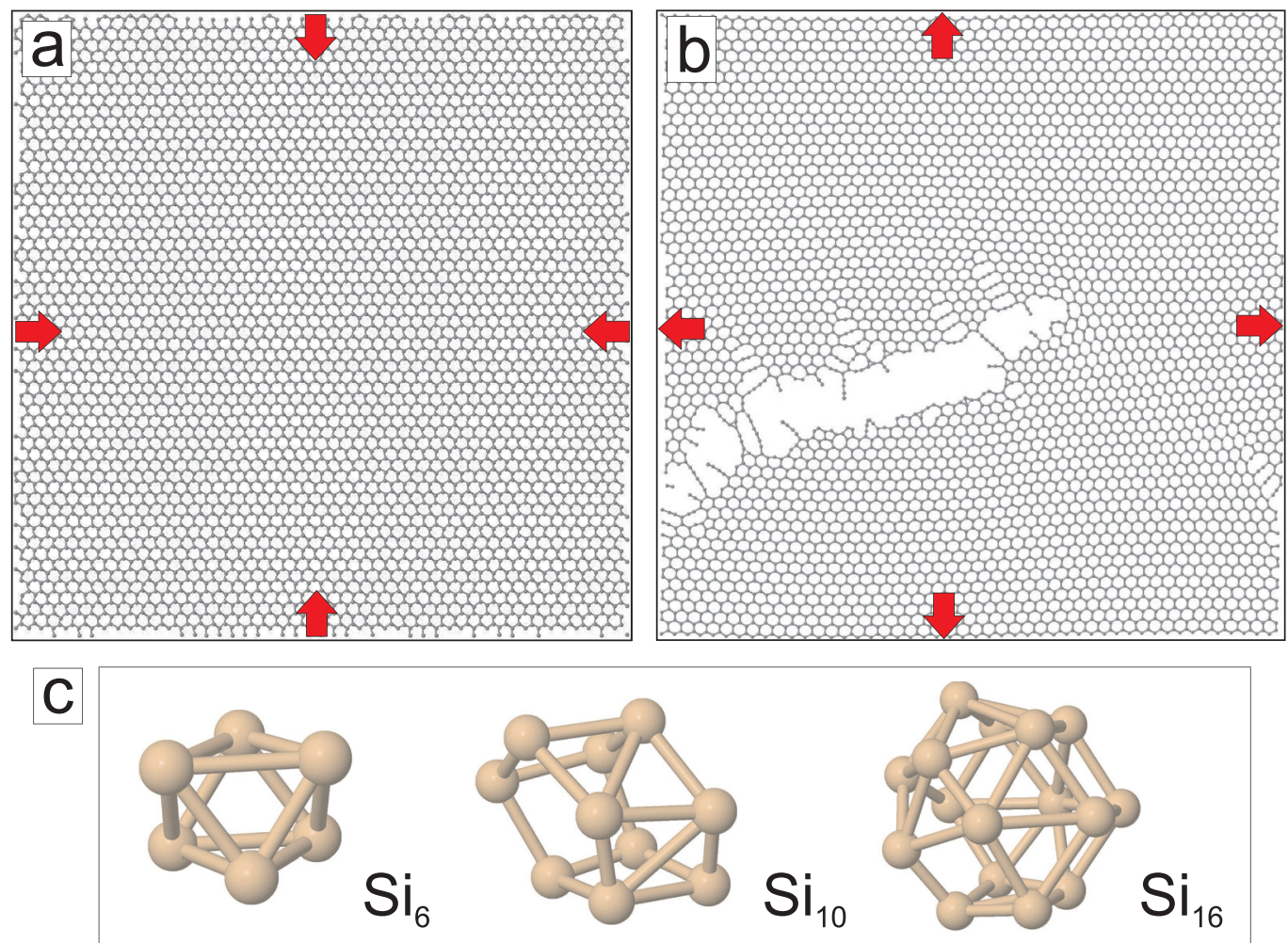

FIG. 1. Graphene sheet under (a) compressive $(-3 \%)$ and (b) tensile (2.5\%) strain. Arrows indicate the direction of the strain. (c) Silicon clusters thermalized at room temperature. 
cluster bombardment, for given parameters of the system (i.e., size and energy of the Si clusters and the strain applied to the graphene), we conducted a statistical analysis, and the results presented in this manuscript are averaged over an ensemble of 5 different initial distributions of velocities of the carbon atoms and Si clusters. All the simulations were carried out using the LAMMPS $\operatorname{code}^{42}$ which includes the ReaxFF force fields. ${ }^{43}$

Nanopores created after the cluster bombardment are characterized as follows. First, we monitored the number of carbon atoms removed from the graphene sheet. Second, we estimated the effective pore diameter measuring the area of the pore $A$ as $d=\sqrt{4 A / \pi}$. To show how regular the pores are, we fit the nanopores with an ellipse and define its "roundness" by $2 A / a \pi$, where $a$ is the semi-major axis of the ellipse. Analyses are conducted using Jmol and ImageJ packages.

\section{COMPRESSIVE STRAIN}

We start by considering Si cluster bombardment of graphene under compressive strain. We limit ourselves to a single and perpendicular impact of Si clusters of different size and energies, i.e., the effect of incidence angle is not investigated. Figures 2(a)-2(d) show snapshots of graphene under $-3 \%$ of compressive strain after the impact of $\mathrm{Si}_{6}$ cluster with different initial energies. For all the considered shooting energies, severe damage of graphene is observed due to irradiation. The defective structures are quite irregular and contain different net-like dangling carbon chains. These carbon chains and the dangling bonds at the edges of the pores will act as reactive centers and affect the properties of graphene for, e.g., water applications. Silicon atoms can also attach to graphene for smaller kinetic energies of the Si clusters (Fig. 2(a)). The resistance of graphene to damage due to the cluster bombardment increases with reducing compressive strain (Figs. 2(e)-2(1)): the defects become more regular and smaller in size. However, the irregularities are still observed with dominant carbon chains. Despite the single impact of the clusters, pores can be formed in different places. Replacement of carbon atoms with $\mathrm{Si}$ atoms occurs at low energies of the clusters (Figs. 2(e) and 2(i)).

To see the effect of the size (i.e., mass) of the clusters on the damage of compressively strained graphene, we show
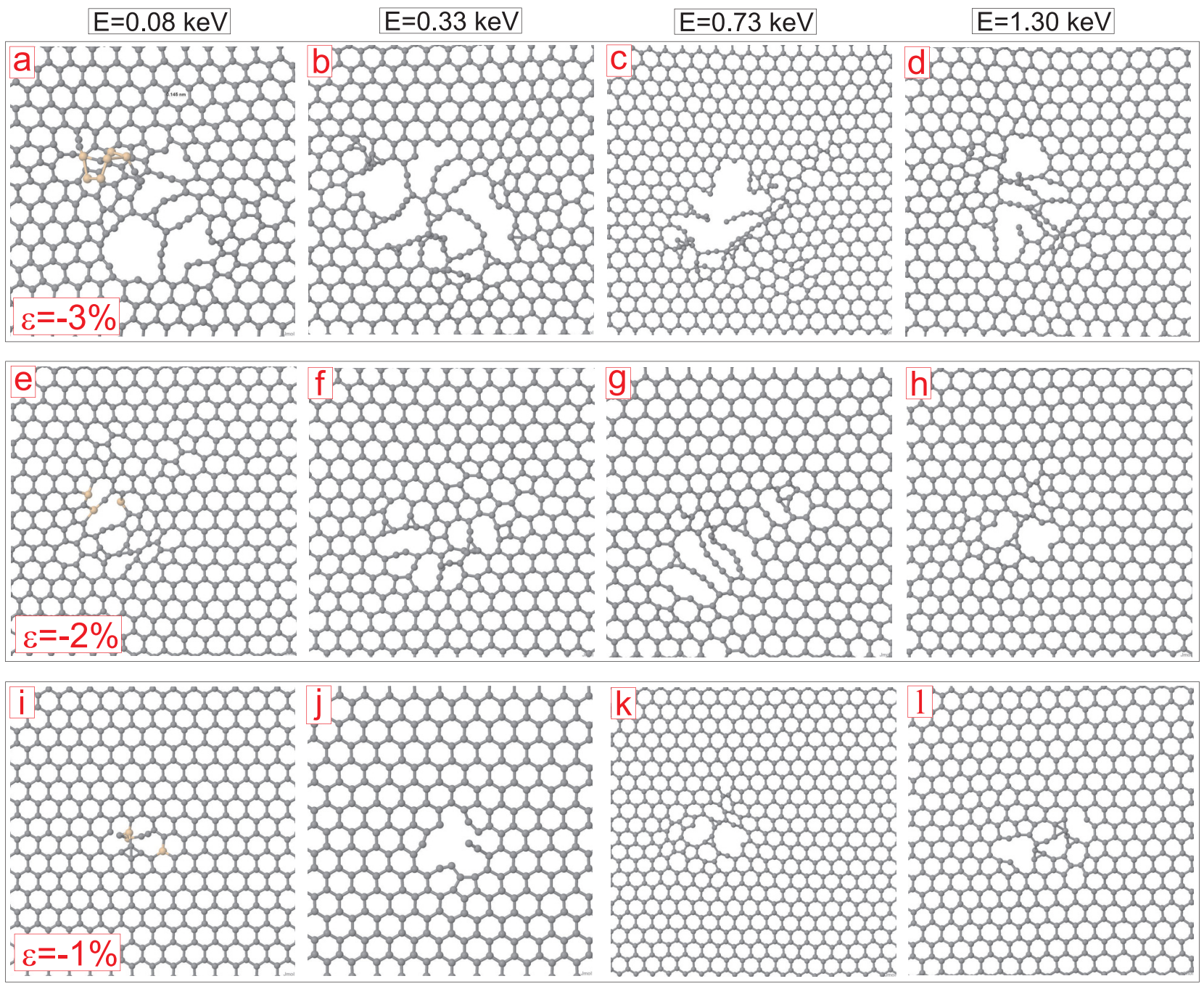

FIG. 2. Typical structures of graphene under compressive strain $\epsilon=-3 \%$ (a)-(d), $\epsilon=-2 \%$ (e)-(h), and $\epsilon=-1 \%$ (i)-(1) after bombardment with $\mathrm{Si}_{6}$ cluster with different energies: $E=13.55 \mathrm{eV} /$ atom (first column), $E=54.2 \mathrm{eV} /$ atom (second column), $E=121.95 \mathrm{eV} /$ atom (third column), and $E=216.8 \mathrm{eV} /$ atom (fourth column). Snapshots are plotted after 200 ps of NVE MD simulations after the impact. 
in Fig. 3 snapshots of graphene under 3\% compressive strain after the impact of $\mathrm{Si}_{6}, \mathrm{Si}_{10}$, and $\mathrm{Si}_{16}$ cluster with different kinetic energies. For the considered value of the strain, the defects are irregular, and the dangling carbon chains dominate the topologies. With increasing size of the clusters, the area of damage increases and defects can appear in different places far from each other. The absorption of $\mathrm{Si}$ atoms becomes more favorable. Note that the position of most of the carbon atoms in the defected area is displaced from the plane of graphene, i.e., 3D structures are obtained.

To characterize the damage of graphene by Si clusters, we plotted in Fig. 4 the number of carbon atoms, $N$, removed from graphene during the bombardment as a function of cluster energy. The number of $\mathrm{Si}$ atoms attached to graphene is not counted. It is seen from this figure that for all sizes of the $\mathrm{Si}$ clusters, the largest removal of carbons atoms increases with increasing compressive strain (see black columns). Since the pores are irregular, we did not attempt to characterize the size and regularities of the created pores.

Thus, compressive strain does not result in improved nanopore creation in graphene and should be avoided during perforation of graphene with nanoparticles.

\section{TENSILE STRAIN}

In this section, we study the effect of tensile strain on the nanopore creation process in graphene by bombardment with Si clusters. Without cluster impact, graphene retains its hexagonal structure up to $2.5 \%$ of tensile strain, which is in good agreement with recent theoretical and experimental reports. ${ }^{44-48}$ When the critical strain is reached, the breakage of graphene starts at a point and spreads to the rest of graphene (see Fig. 1(b)). Note that the position and the size of the initial voids cannot be controlled in such simulations. In addition, voids expand in time resulting in the failure of graphene.

As a reference, we also study nanopore creation in unstrained graphene, snapshots of which are shown in Figs. 5(a)-5(d), after the impact of $\mathrm{Si}_{6}$ cluster with different energies. Depending on the shooting energy, we observe the following processes: (i) total reflection at small energies $E<10 \mathrm{eV}$ with no damage in graphene (see supplementary material); (ii) absorption of $\mathrm{Si}$ atoms with consequent damage of graphene $10 \mathrm{eV}<E<150 \mathrm{eV}$ (see Fig. 5(a) and supplementary material); and (iii) transmission followed by vacancy formation $E>150 \mathrm{eV}$ (see Figs. 5(b)-5(d) and
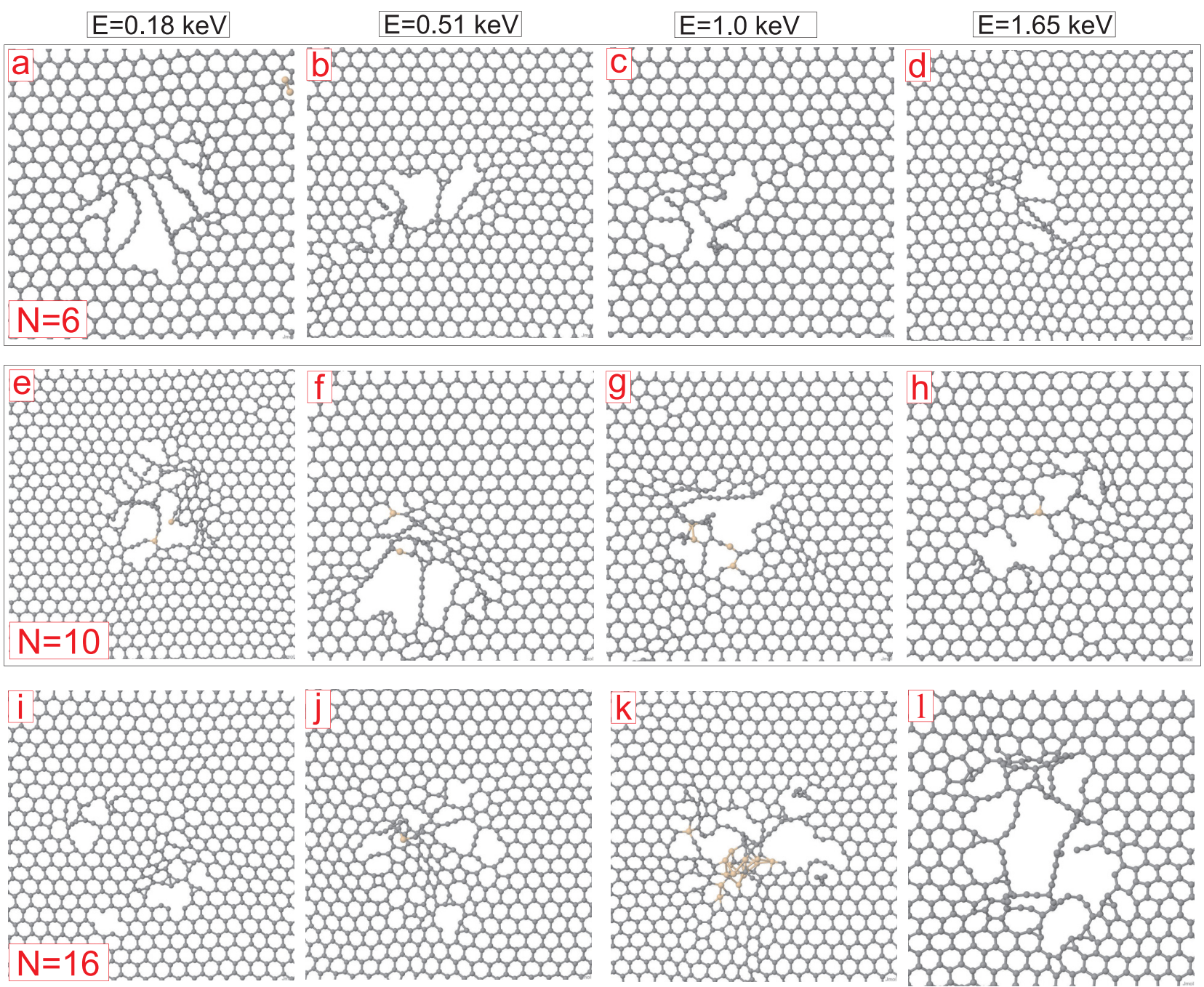

FIG. 3. Topology of graphene under $\epsilon=-3 \%$ strain after the impact of $\mathrm{Si}_{6}(\mathrm{a})-(\mathrm{d}), \mathrm{Si}_{10}(\mathrm{e})-(\mathrm{h})$, and $\mathrm{Si}_{16}$ (i)-(l). Energies of the $\mathrm{Si}$ clusters per atom are shown on top of each column. Results are shown after 200 ps of simulation time. 


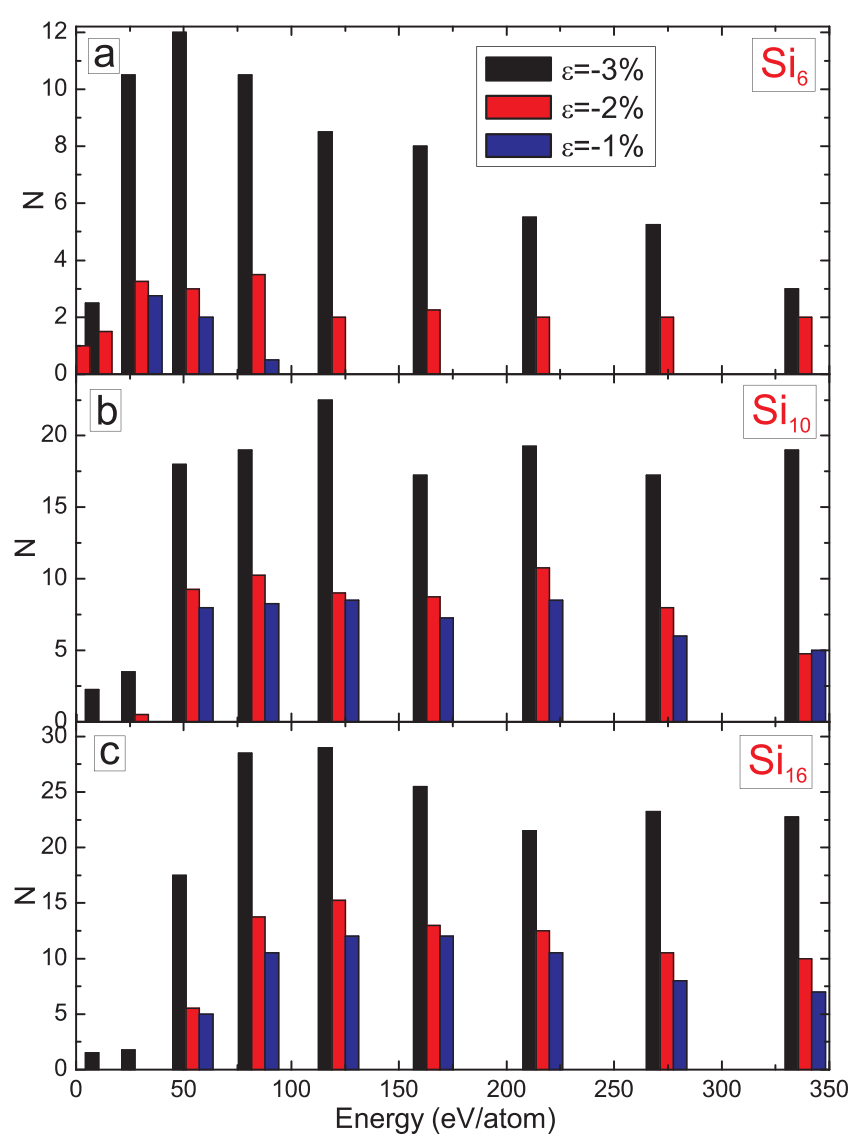

FIG. 4. Number of carbon atoms removed from graphene that is under tensile strain $\epsilon=-3 \%$ (black columns), $\epsilon=-2 \%$ (red columns), and $\epsilon=-1 \%$ (blue columns) as a function of the energy per atom of the clusters: $\mathrm{Si}_{6}$ (a), $\mathrm{Si}_{10}(\mathrm{~b})$, and $\mathrm{Si}_{16}$ (c).

supplementary material). These numbers are in agreement with the recent reports by Qin et al. ${ }^{30}$ Small difference in the energy intervals could be due to the larger size of the clusters in our simulations. For the considered sizes of the clusters, we did not observe transmission of the Si clusters through graphene without creating nanopores. The created nanopores have smoother edges as compared to the case of compressive strain (see Fig. 2). Although long carbon chains become less dominant in the obtained structures, some short carbon chains still exist in the system (Figs. 7(b)-7(d)). Note that we presented the graphene structures 200 ps after the impact. Therefore, defect structures are different from the initial ones due to the elastic response of graphene leading to efficient closure of the nanopores.

Figures 5(e)-5(p) show snapshots of tensile strained graphene after $\mathrm{Si}_{6}$ cluster interaction. The strain decreases the elastic response of graphene to the $\mathrm{Si}$ clusters, and the absorption of the Si atoms becomes less pronounced. Most importantly, for the same energy of the Si clusters, the nanopores become larger in size and their edges are smoother. However, the clusters reduce the fracture strength of graphene significantly - fracturing starts already at $\varepsilon \geq 2 \%$ (see Figs. 5(r) and 5(t)). The reason is that the impact of clusters can initiate fracturing. Net-like defective structures with dominating dangling carbon atom chains do not appear under tensile strain. Structural integrity of graphene remains during the bombardment up to $\varepsilon=2 \%$. Similar results were obtained for larger sizes of the Si clusters (see Figs. 6 and 7). At smaller shooting energies, the dominant process is absorption (panels (a), (e), (i), (m), and (q) in Figs. 6 and 7) with almost all the $\mathrm{Si}$ atoms in the cluster absorbed by the graphene; nanopores become more circular with increasing strain and fracturing becomes more pronounced at larger values of $\varepsilon$ (see panels (r) and (s) in Figs. 6 and 7). In addition, passivation of some dangling bonds in the damaged area is also possible (see Figs. 7(f), 7(n), and 7(r)), which increases the stability of the pores. ${ }^{26}$ Except for passivation, the system is free of Si contamination after the impact.

Figure 8 shows the number of carbon atoms $N$ sputtered from graphene under tensile strain as a function of the energy of Si clusters of different sizes. For smaller size clusters (Fig. 8(a)), removal of carbon atoms occurs only for smaller energies of the clusters $(E<120 \mathrm{eV} /$ atom $)$ with the averaged maximum number lacking carbon atoms not exceeding $\mathrm{N}=3$. The number of displaced carbon atoms decreases with increasing cluster energy, which is in good agreement with previous reports. ${ }^{32,49}$ Figures $8(\mathrm{~b})$ and $8(\mathrm{c})$ show the results obtained for $\mathrm{Si}_{10}$ and $\mathrm{Si}_{16}$ cluster bombardment. As expected, more carbon atoms are removed from graphene with increasing size of the cluster. For smaller energies of the $\mathrm{Si}$ clusters $(E<100 \mathrm{eV} /$ atom $)$, the tensile strain increases the number of removed carbon atoms. However, $\mathrm{N}$ does not strongly depend on the strength of the applied strain; similar number of carbon atoms is removed for cluster energies $E>100 \mathrm{eV} /$ atoms.

The surface area $A$ of the created nanopores strongly depends on the applied strain. This is shown in Fig. 9, where we plot $A$ as a function of Si cluster energy for all three considered samples. Depending on the value of $\varepsilon$, the surface area can increase by more than twice. The effect is more pronounced for larger energies of the clusters. Note that defect structures can be created even without the removal of carbon atoms from graphene (compare Figs. 8(a) and 9(a)), i.e., nanopores are created not because of the loss of carbon atoms but due to the rearrangement of carbon atoms in the impact area. The surface area of the nanopores is directly proportional to the size of the clusters. Larger size of the nanopores in strained graphene is due to the reduced elasticity of graphene which prevents the closure of the pores after the bombardment. It is seen from Figs. 9(b) and 9(c) that using clusters with more than $10 \mathrm{Si}$ atoms and with energies in the range between 50 and $350 \mathrm{eV} /$ atom enables one to create nanopores of approximately $1 \mathrm{~nm}^{2}$ surface area.

To characterize the created nanopores and to find the relation between the nanopore topology and incident energy/ strain, we fitted each of the pores with an ellipse and calculated its roundness by $R=2 A / a \pi$, where $a$ is the semi-major axis of the ellipse. Figure 10 shows the roundness of the nanopores as a function of energy of the Si clusters. At small shooting energies $E \leq 500 \mathrm{eV}$, similar roundness is obtained for both unstrained and strained samples. However, the nanopores become more circular with increasing external strain. This is in addition to the fact that the size of the nanopores also increases with increasing strain (see Fig. 9). 

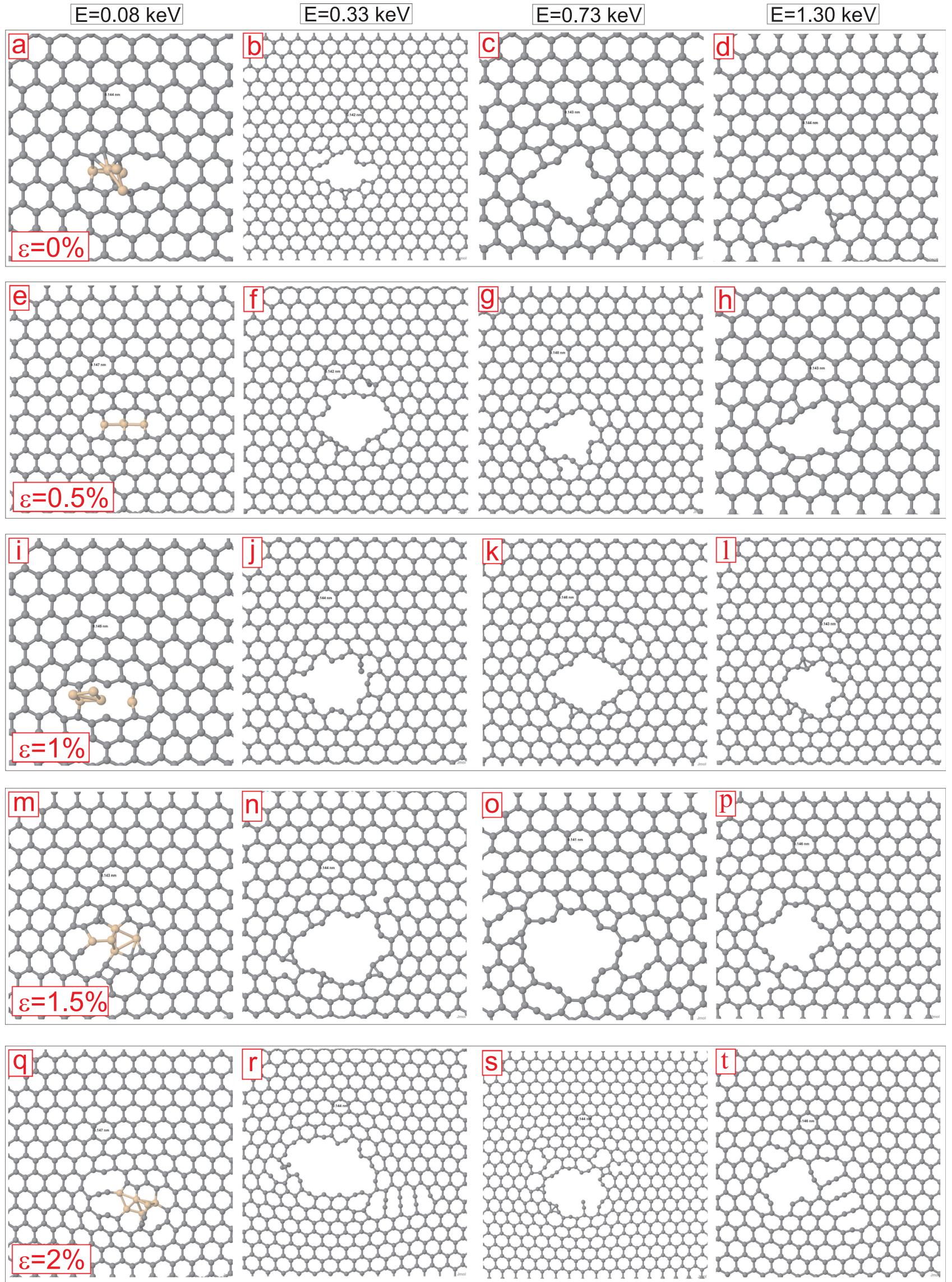

FIG. 5. Structures of graphene under tensile strain $\epsilon=0 \%$ (a)-(d), $\epsilon=0.5 \%$ (e)-(h), $\epsilon=1 \%$ (i)-(l), $\epsilon=1.5 \%$ (m)-(p), and $\epsilon=2 \%$ (q)-(t) after bombardment with $\mathrm{Si}_{6}$ cluster. The energies of the cluster are $E=13.55 \mathrm{eV} /$ atom (first column), $E=54.2 \mathrm{eV} /$ atom (second column), $E=121.95 \mathrm{eV} /$ atom (third column), and $E=216.8 \mathrm{eV} /$ atom (fourth column). Snapshots are plotted after 200 ps of NVE MD simulations after the impact. 

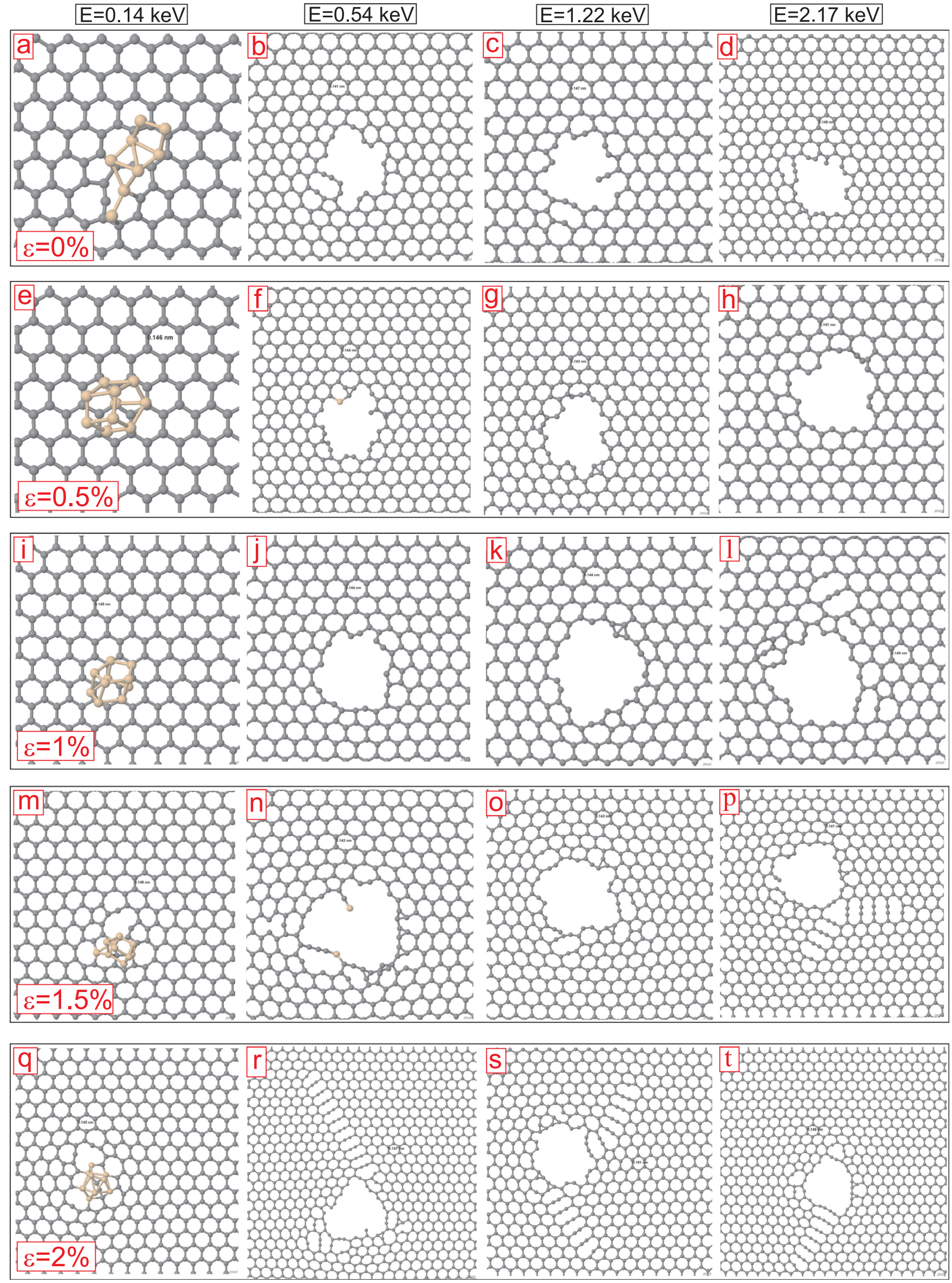

FIG. 6. The same as Fig. 5 but here for $\mathrm{Si}_{10}$ clusters. 

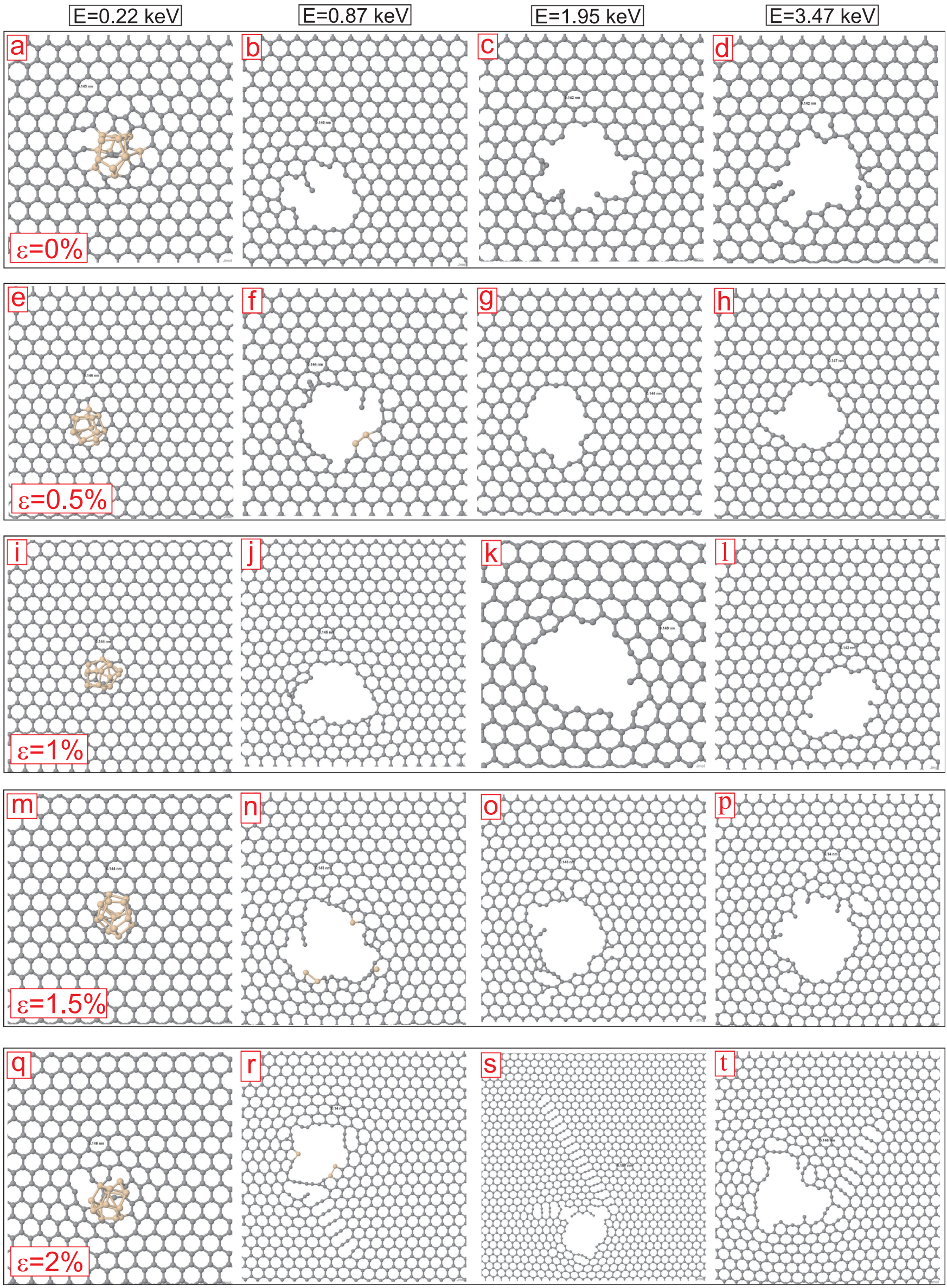

FIG. 7. The same as Fig. 5 but here for $\mathrm{Si}_{16}$ clusters. 


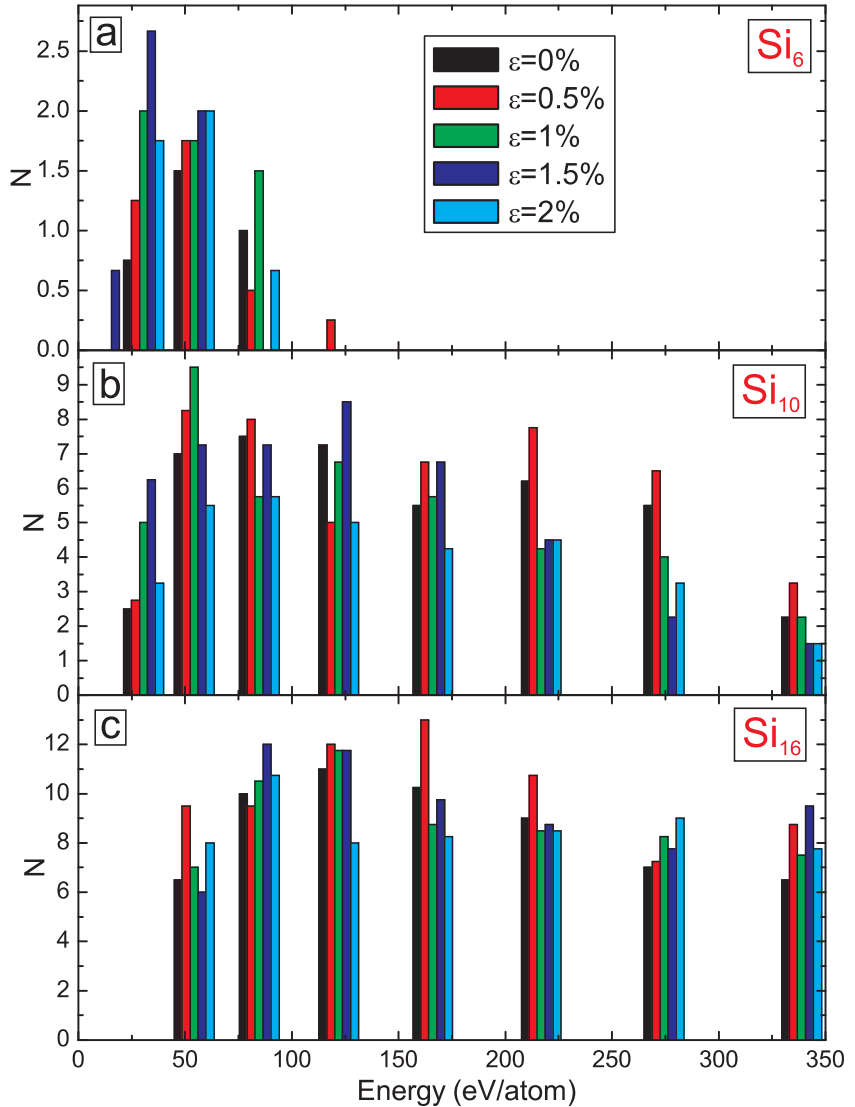

FIG. 8. Number of carbon atoms removed from graphene under tensile strain as a function of energy of the $\mathrm{Si}$ clusters: $\mathrm{Si}_{6}$ (a), $\mathrm{Si}_{10}$ (b), and $\mathrm{Si}_{16}$ (c).

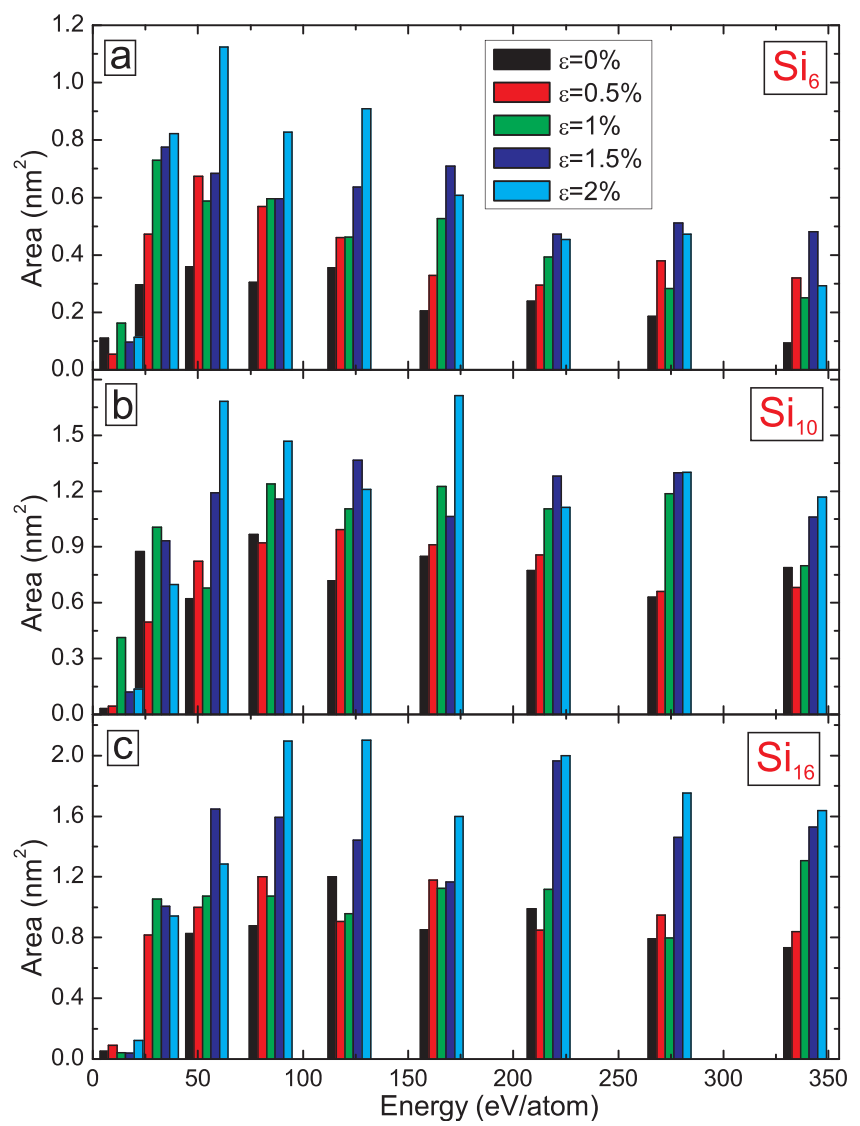

FIG. 9. Area of nanopores created in graphene after the bombardment with $\mathrm{Si}_{6}(\mathrm{a}), \mathrm{Si}_{10}$ (b), and $\mathrm{Si}_{16}$ (c) clusters with different energies.

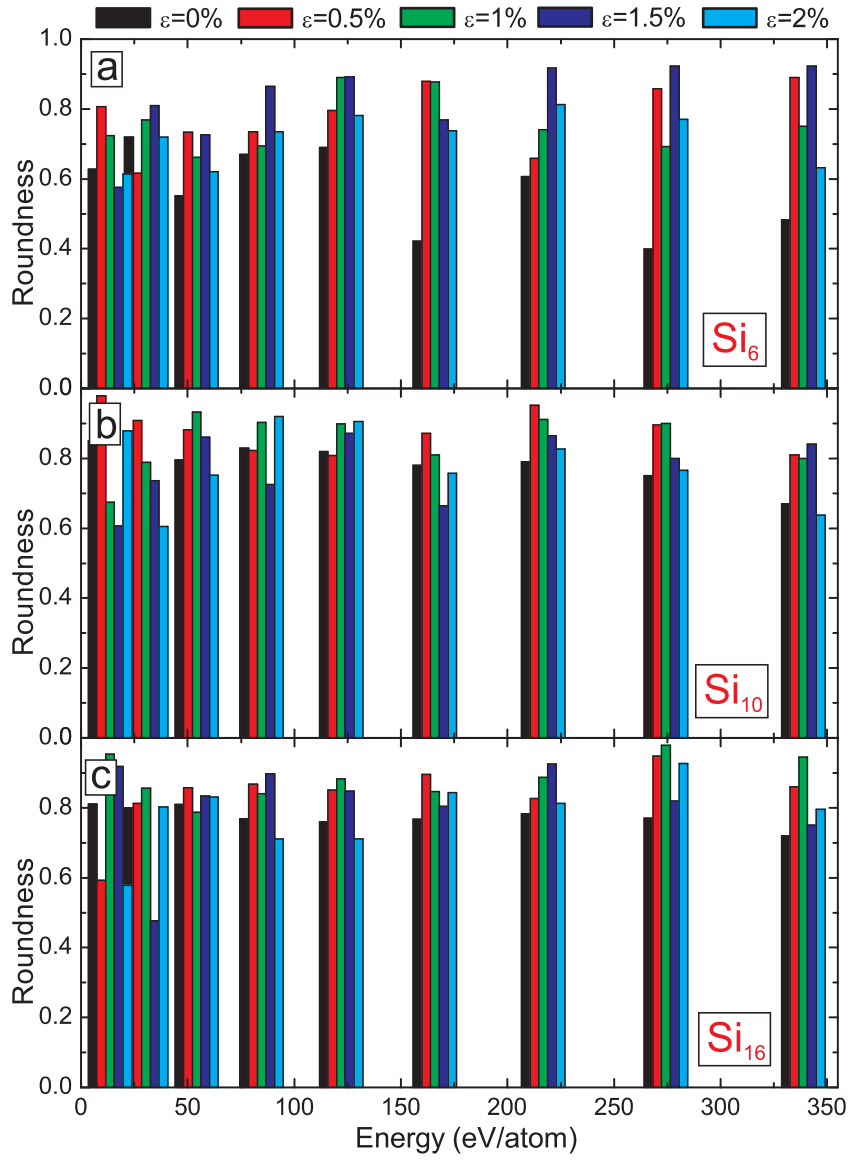

FIG. 10. Regularity of nanopores created in graphene after the bombardment with $\mathrm{Si}_{6}(\mathrm{a}), \mathrm{Si}_{10}$ (b), and $\mathrm{Si}_{16}$ (c) clusters with different energies.

Thus, external strain enables one to obtain more regular nanopores in graphene during cluster bombardment. The kinetic energy of the interacting clusters also plays an important role in this process and should be taken into account in experiments.

\section{CONCLUSIONS}

Using reactive molecular dynamics simulations, we studied the interaction of $\mathrm{Si}$ clusters with graphene under external strain. Compressive strain reduces the resistance of graphene to the bombardment: severe radiation damage is produced with cluster having large energy with the creation of net-like dangling carbon chains. Such topologies are not desirable for applications of graphene in the area of separation (i.e., desalination). On the contrary, tensile strain results in the creation of nanopores with more regular shape and smooth edges. The cluster size and incident energy also play an important role in the creation process of nanopores (i.e., reflection, absorption, and damage). Our results show the positive effect of external tensile strain for the controlled nanopore creation in graphene during cluster bombardment.

\section{SUPPLEMENTARY MATERIAL}

See supplementary material for the bombardment of unstrained graphene (960 carbon atoms in the simulation 
cell) with a $\mathrm{Si}_{6}$ cluster with energies $20 \mathrm{eV}, 80 \mathrm{eV}$, and $330 \mathrm{eV}$.

\section{ACKNOWLEDGMENTS}

Computational resources were provided by the HPC core facility CalcUA at the University of Antwerp.

${ }^{1}$ K. S. Novoselov, A. K. Geim, S. V. Morozov, D. Jiang, M. I. Katsnelson, I. V. Grigorieva, S. V. Dubonos, and A. A. Firsov, Nature (London) 438 197 (2005).

${ }^{2}$ K. S. Novoselov, A. K. Geim, S. V. Morozov, D. Jiang, Y. Zhang, S. V. Dubonos, I. V. Grigorieva, and A. A. Firsov, Science 306, 666 (2004).

${ }^{3}$ A. K. Geim and K. S. Novoselov, Nat. Mater. 6, 183 (2007).

${ }^{4}$ A. K. Geim, Science 324, 1530 (2009).

${ }^{5}$ X. Wang, X. Li, L. Zhang, Y. Yoon, P. K. Weber, H. Wang, J. Guo, and H. Dai, Science 324, 768 (2009).

${ }^{6}$ F. Schedin, A. K. Geim, S. V. Morozov, E. W. Hill, P. Blake, M. I. Katsnelson, and K. S. Novoselov, Nat. Mater. 6, 652 (2007).

${ }^{7}$ L. Qiu, X. Zhang, W. Yang, Y. Wang, G. P. Simon, and D. Li, Chem. Commun. 47, 5810 (2011).

${ }^{8}$ R. R. Nair, H. A. Wu, P. N. Jayaram, I. V. Grigorieva, and A. K. Geim, Science 335, 442 (2012).

${ }^{9}$ D. Cohen-Tanugi and J. C. Grossman, Nano Lett. 12, 3602 (2012).

${ }^{10}$ M. Hu and B. Mi, Environ. Sci. Technol. 47, 3715 (2013).

${ }^{11}$ D. W. Boukhvalov, M. I. Katsnelson, and Y.-W. Son, Nano Lett. 13, 3930 (2013).

${ }^{12}$ S. C. O’Hern, M. S. H. Boutilier, J.-C. Idrobo, Y. Song, J. Kong, T. Laoui, M. Atieh, and R. Karnik, Nano Lett. 14, 1234 (2014).

${ }^{13}$ S. P. Surwade, S. N. Smirnov, I. V. Vlassiouk, R. R. Unocic, G. M. Veith, S. Dai, and S. M. Mahurin, Nat. Nanotechnol. 10, 459 (2015).

${ }^{14}$ A. Subramani and J. G. Jacangelo, Water Res. 75, 164 (2015).

${ }^{15}$ S. Daer, J. Kharraz, A. Giwa, and S. W. Hasan, Desalination 367, 37 (2015).

${ }^{16}$ A. Aghigh, V. Alizadeh, H. Y. Wong, Md. S. Islam, N. Amin, and M. Zaman, Desalination 365, 389 (2015).

${ }^{17}$ S. Dervin, D. D. Dionysiouc, and S. C. Pillai, Nanoscale 8, 15115 (2016).

${ }^{18}$ H. W. Yoon, Y. H. Cho, and H. B. Park, Philos. Trans. R. Soc. A 374, 20150024 (2016).

${ }^{19}$ K. Goh, H. E. Karahan, L. Wei, T.-H. Bae, A. G. Fane, R. Wang, and Y. Chen, Carbon 109, 694 (2016).

${ }^{20}$ D. Cohen-Tanugi and J. C. Grossman, Desalination 366, 59 (2015).

${ }^{21}$ T. Jain, B. C. Rasera, R. J. S. Guerrero, M. S. H. Boutilier, S. C. O'Hern, J.-C. Idrobo, and R. Karnik, Nat. Nanotechnol. 10, 1053 (2015).

${ }^{22}$ O. Leenaerts, B. Partoens, and F. M. Peeters, Phys. Rev. B 77, 125416 (2008).
${ }^{23}$ W. Yuan, J. Chen, and G. Shi, Mater. Today 17, 77 (2014).

${ }^{24}$ M. Tagliazucchi and I. Szleifer, Mater. Today 18, 131 (2015).

${ }^{25}$ K. A. Mahmoud, B. Mansoor, A. Mansour, and M. Khraisheh, Desalination 356, 208 (2015).

${ }^{26}$ J. Lee, Z. Yang, W. Zhou, S. J. Pennycook, S. T. Pantelides, and M. F. Chisholm, PNAS 111, 7522 (2014).

${ }^{27}$ M. Becton, L. Zhang, and X. Wang, J. Nanomech. Micromech. 4, B4014002 (2014).

${ }^{28}$ H. Wang, Q. Wang, Y. Cheng, K. Li, Y. Yao, Q. Zhang, C. Dong, P. Wang, U. Schwingenschlogl, W. Yang, and X. X. Zhang, Nano Lett. 12, 141 (2012).

${ }^{29}$ S. Zhao, J. Xue, L. Liang, Y. Wang, and S. Yan, J. Phys. Chem. C 116, 11776 (2012).

${ }^{30}$ X.-M. Qin, T.-H. Gao, W.-J. Yan, X.-T. Guo, and Q. Xie, J. Mol. Struct. 1061, 19 (2014).

${ }^{31}$ E. P. Bellido and J. M. Seminario, "Molecular dynamics simulations of ion-bombarded graphene," J. Phys. Chem. C 116, 4044 (2012).

${ }^{32}$ W. Li, L. Liang, S. Zhao, S. Zhang, and J. Xue, J. Appl. Phys. 114, 234304 (2013).

${ }^{33}$ Z. Bai, L. Zhang, H. Li, and L. Liu, ACS Appl. Mater. Interfaces 8, 24803 (2016).

${ }^{34}$ H. Wang, K. Kurata, T. Fukunaga, H. Takamatsu, X. Zhang, T. Ikuta, K. Takahashi, T. Nishiyama, H. Ago, and Y. Takata, Carbon 99, 564 (2016).

${ }^{35}$ J. Azamat, J. Phys. Chem. C 120, 23883 (2016).

${ }^{36}$ D. Cohen-Tanugi, L.-C. Lin, and J. C. Grossman, Nano Lett. 16, 1027 (2016).

${ }^{37}$ S. Standop, O. Lehtinen, C. Herbig, G. Lewes-Malandrakis, F. Craes, J. Kotakoski, T. Michely, A. V. Krasheninnikov, and C. Busse, Nano Lett. 13, 1948 (2013)

${ }^{38}$ X. Wu, H. Zhao, and J. Pei, Comput. Mater. Sci. 102, 258 (2015).

${ }^{39}$ S. Zhao and J. Xue, Carbon 93, 169 (2015).

${ }^{40}$ A. C. T. van Duin, S. Dasgupta, F. Lorant, and W. A. Goddard, J. Phys. Chem. A 105, 9396 (2001).

${ }^{41}$ G. R. Berdiyorov, M. Neek-Amal, F. M. Peeters, and A. C. T. van Duin, Phys. Rev. B 89, 024107 (2014).

${ }^{42}$ G. Kresse and J. Furthmuller, Phys. Rev. B 54, 11169 (1996).

${ }^{43}$ S. V. Zybin, W. A. Goddard, P. Xu, A. C. T. van Duin, and A. P. Thompson, Appl. Phys. Lett. 96, 081918 (2010).

${ }^{44}$ B. Mortazavi and S. Ahzi, Solid State Commun. 152, 1503 (2012).

${ }^{45}$ B. Mortazavi, Y. Rémond, S. Ahzi, and V. Toniazzo, Comput. Mater. Sci. 53, 298 (2012).

${ }^{46}$ B. Mortazavi and G. Cuniberti, Nanotechnology 25, 215704 (2014).

${ }^{47}$ B. Mortazavi, Z. Fan, L. F. C. Pereira, A. Harju, and T. Rabczuk, Carbon 103, 318 (2016)

${ }^{48}$ D. S. Kim, V. Dhand, K. Y. Rhee, and S.-J. Park, Polymers 7, 527 (2015).

${ }^{49}$ D. C. Pujals, Y. A. Corrales, and F. Baldassarre, J. Radioanal. Nucl. Chem. 289, 167 (2011). 